Pacific Journal of Mathematics

THE SOLUTION OF SINGULAR EQUATIONS, I. LINEAR
EQUATIONS IN HILBERT SPACE 


\title{
THE SOLUTION OF SINGULAR EQUATIONS, I: LINEAR EQUATIONS IN HILBERT SPACE
}

\author{
Thomas I. SeIdman
}

\begin{abstract}
The theorem on which this paper is based is an easy generalization of the fact that the nullspace of an operator is the orthocomplement of (the closure of) the range of its adjoint. Its significance, here, is the observation that this may be applied to give a computationally feasible algorithm for the problem of the title.
\end{abstract}

Consider a pair of hilbert spaces $U, V$ and a linear transformation $A: U \rightarrow V$. For any $b$ in the range of $A$ there is, by definition, a solution of the equation:

$$
A x=b
$$

It is specifically not assumed here that the range of $A$ is closed in $V$ so, in general, the solution cannot be taken to depend continuously on $b$. Since, in many contexts, the inhomogeneous term $b$ is known only through 'measurement' — hence, only to 'arbitrary but finite' accuracy_- this lack of continuous dependence has heretofore been taken to preclude useful computation; see, e.g., Hadamard's discussion of a 'well-posed' problem [4]. There has been, however, considerable interest (some references are noted in $\S 5$ ) in computational approaches to various problems which are ill-posed in Hadamard's sense. Even for well-posed problems it has proved desirable to distinguish in principle (cf. [9], p. 224) between the notions of solvability (existence) and approximation-solvability (obtaining a solution - granting solvability - as a limit of solutions of finite dimensional problems).

Numerous examples of such singular situations as we consider might easily be adduced at this point: the backward heat equation, integral equations of the first kind, analytic continuation, inversion of the Laplace transform, etc. The particular application through which the author came to the algorithm described here concerned synthesis of a boundary null-control for the heat equation. This has already been discussed in greater detail elsewhere [2] but will be treated briefly here $\S 6$ as an example. The author would like to dedicate this paper to the memory of W. C. Chewning, who initiated that work, with thanks and in regret for his untimely death.

2. Basic theorem. We consider the following general setting: 
- a pair of hilbert spaces $U, V$,

- a densely defined linear map $A: D \subset U \rightarrow V$,

- an element $b \in R(A)$ so $b=A x_{*}$ for some $x_{*} \in D$,

- a subspace $Y$ of $D_{*}$ (the domain of the adjoint $A^{*}$ ).

We let $X$ be the closure in $U$ of $A^{*} Y$ and let

$$
S=\{x \in D:(A x-b) \perp Y\}, S^{\prime}=\left\{x \in U:\left\langle A^{*} y, x\right\rangle=\langle y, b\rangle \text { for } y \in Y\right\} \text {. }
$$

Let $P$ be the orthoprojection onto $X$.

REMARKS. (1) It is important for the argument below that $U$ be a hilbert space but, with minor (notational) modifications, $V$ may be more general. A further generalization of the algorithm to be presented applies when $U$ is also more general (a uniformly convex banach space) but this requires a more subtle convergence argument and will be discussed elsewhere [12].

(2) If, as is the case in each of the examples adduced above, the transformation $A$ is continuous, one has $D=U$ and $D_{*}=V^{*}(=V)$ so the restriction: $Y \subset D_{*}$ is automatically satisfied, as is the condition: $X \subset D$ which appears in the Theorem below.

THeOREM I. Let the setting be as described above. Then the following are equivalent:

(i) $\hat{x}=P x_{*}$,

(ii) $\hat{x}$ is the nearest point in $X$ to the solution $x_{*}$,

(iii) $\hat{x} \in X \cap S^{\prime}$,

(iv) $\hat{x}$ has minimal norm in $S^{\prime}$.

If $X \subset D$, then $S^{\prime}$ may be replaced by $S$ in (iii), (iv).

Proof. The equivalence of (i), (ii) is standard. Observe that $x_{*} \in S \subset S^{\prime}$ and that $S^{\prime}$ is a translate (e.g., by $x_{*}$ ) of the closed subspace $X^{\perp}\left(=\left(A^{*} Y\right)^{\perp}\right.$ since $A^{*} Y$ is dense in $\left.X\right)$. Thus,

$$
S^{\prime}=x_{*}+X^{\perp}, \quad S=x_{*}+\left(X^{\perp} \cap D\right) .
$$

It follows that $\hat{x} \in X \cap S^{\prime}$ implies $\hat{x}=P \hat{x}=P x_{*}$ so (iii) implies (i); conversely, $P \hat{x}=\hat{x}=P x_{*}$ implies $\hat{x} \in S^{\prime} \cap X$. This also shows that (iii) determines a unique point $x_{3}$ (i.e., $S^{\prime} \cap X=\left\{x_{3}\right\}$ ); if $X \subset D$, then $x_{3} \in D$ and $X \subset S^{\prime}=X \cap S$. Now (3) implies that the minimum of $\|x\|$ for $x \in S^{\prime}$ is attained for $x=x_{3} \in X$ so (iii), (iv) are equivalent. If $X \subset D$, this also shows that $\|x\|$ actually attains a minimum in $S$ even though $S$ need not be closed.

3. Finite dimensional subspaces. The cases of practical (com- 
putational) importance involve finite dimensional subspaces: $Y=$ $s p\left\{y_{1}, \cdots, y_{n}\right\}$ so $X=s p\left\{z_{1}, \cdots, z_{n}\right\}$ with $z_{j}=A^{*} y_{j}$ for each $j$.

It will be apparent that there is no loss of generality in assuming $Y$ is such that $\left\{z_{1}, \cdots, z_{n}\right\}$ is linearly independent: i.e., the restriction of $A^{*}$ to $Y$ is nonsingular. It is now computationally easiest to work with the characterization (iii) of the Theorem - which here reduces to the finite set of conditions for $\hat{x} \in X$ :

$$
\left\langle z_{j}, \hat{x}\right\rangle=\left\langle y_{j}, b\right\rangle \text { for } j=1, \cdots, n .
$$

Note that if each $z_{j}$ is in $D$ (each $y_{j}$ in the domain of $A A^{*}$ ) so $X \subset D$, then (4) is equivalent to:

$$
\left\langle y_{j}, A \hat{x}-b\right\rangle=0 \text { for } j=1, \cdots, n \text {. }
$$

Expanding $\hat{x} \in X$ with respect to the basis $\left\{z_{1}, \cdots, z_{n}\right\}$ (i.e., setting $\hat{x}=\xi_{1} z_{1}+\cdots+\xi_{n} z_{n}$ ) the conditions (4) provide $n$ linear equations for the $n$ unknown coefficients:

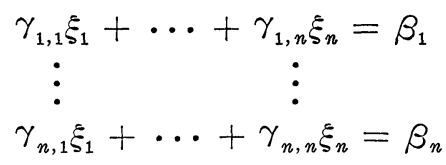

where

$$
\begin{aligned}
& \gamma_{j, k}=\left\langle z_{j}, z_{k}\right\rangle \quad(j, k=1, \cdots n), \\
& \beta_{j}=\left\langle j_{j}, b\right\rangle \quad(j=1, \cdots, n) .
\end{aligned}
$$

Thus the matrix $G=\left(\left(\gamma_{j, k}\right)\right)$ is the Gramian matrix for the basis $\left\{z_{1}, \cdots, z_{n}\right\}$ of $X$ and is clearly nonsingular.

From the nature of the formulation (5), (6) it is clear that the point $\hat{x}$ determined thereby will be stable under small perturbations of the data and computational imprecisions even though the original transformation $A$ may be singular. Indeed, it is this consideration which makes the method a useful approach to singular problems.

4. Solution of singular problems. In applying the discussion above to the computational solution of a (possibly singular) problem of the form (1), we consider the following general setting:

- a pair of hilbert spaces $U, V$,

- a densely defined linear map $A: D \subset U \rightarrow V$,

- an element $b \in R(A)$ so $b=A x_{*}$ for some $x_{*} \in D$,

- a sequence $\left\{y_{1}, y_{2}, \cdots\right\}$ in the domain of $A^{*}$.

For $j=1=1,2, \ldots$ we let $z_{j}=A^{*} y_{j}$ and for each $n=1,2 \ldots$ we 
let $Y_{n}=s p\left\{y_{1}, \cdots, y_{n}\right\}, X_{n}=s p\left\{z_{1}, \cdots, z_{n}\right\}$ and define $x_{n}$ as the (unique) solution in $X_{n}$ of the conditions (4).

THeorem II. Let the setting be as above and suppose that $A$ is closed and that $A,\left\{y_{1}, y_{2}, \cdots\right\}$ are such that $N(A) \subset \overline{s p}\left\{z_{1}, z_{2}, \cdots\right\}^{\perp}$ so:

$$
\left\langle z_{j}, x\right\rangle=0(j=1,2, \cdots) \Longrightarrow x \in N(A) .
$$

Then:

(i) for each $n=1,2, \cdots$ the conditions (4) determine a unique $x_{n} \in X_{n}$,

(ii) the sequence $\left(x_{1}, x_{2}, \cdots\right)$ so defined converges to a limit, $\hat{x}$,

(iii) the limit $\hat{x}$ is in $D$ and satisfies (1) $A \hat{x}=b$. If $N(A)$ is nontrivial, $\hat{x}$ will be the unique solution of (1) having minimum norm.

REMARK. Observe that if $A$ is not only closed but continuous, then the condition: $\left\{y_{j}\right\}$ in $D_{*}$ is automatic and (7) is equivalent to requiring that $\operatorname{sp}\left\{N\left(A^{*}\right), y_{1}, y_{2}, \cdots\right\}$ be dense in $V($ e.g., one might take $\left\{y_{1}, y_{2}, \cdots\right\}$ to be any (orthonormal) basis for $V$ ) so $s p\left\{z_{1}, z_{2}, \cdots\right\}$ is dense in $\overline{R\left(A^{*}\right)}$.

Proof (of Theorem II). The unique determination of each $x_{n}$ by (4) follows from Theorem I and the discussion of the finite dimensional case. Let $Y$ be the closure in $V$ of $\mathrm{U}_{n} Y_{n}$ (so $\left.Y=\overline{s p}\left\{y_{1}, y_{2}, \cdots\right\}\right)$ and let $X$ be the closure in $U$ of $\mathrm{U}_{n} X_{n}$ (so $\left.X=s p\left\{z_{1}, z_{2}, \cdots\right\}\right)$. It follows from Theorem I that there is a unique point $\widehat{x}=P x_{*}(P$ is the orthoprojection onto $X$ ) satisfying: $\left\langle A^{*} y, x\right\rangle=\langle y, b\rangle$ for all $y \in Y$ and so, in particular, satisfying (4) for $j=1,2, \ldots$ Setting $x=\left(\hat{x}-x_{*}\right)$, one has

$$
\left\langle z_{j}, x\right\rangle=\left\langle A^{*} y_{j}, \hat{x}\right\rangle-\left\langle y_{j}, A x_{*}\right\rangle=0 \quad(j=1,2, \cdots)
$$

so, from (7), $\left(\hat{x}-x_{*}\right)=x \in N(A) \subset D$. Since $x_{*} \in D$, this means $\hat{x} \in D$ and $A \hat{x}=A x_{*}=b$. The characterization of $\widehat{x}$ when $N(A) \neq\{0\}$ follows from the characterization (iv) of Theorem I. From the characterization (ii) in Theorem I, it follows that $x_{n}$ is the nearest point in $X_{n}$ to $\hat{x}$ (we may now take $x_{*}=\hat{x}$ ), so, as $\bigcup_{n} X_{n}$ is dense in $N(A)^{\perp}$, one must have $x_{n} \rightarrow \widehat{x}$.

5. Discussion. A variety of methods have been applied to the computational solution of ill-posed problems. We mention, in particular, the papers of Krasnoselskii [5] and Kryanev [6], Tikhonov 'regularization' method [13], [3], the work of Nashed and Wahba [8], and the methods of Carasso [1] and of Lattes and Lions [7] for 
backward evolution equations. In addition, even in the absence of theoretical justification there has been extensive use, in computational practice, of 'standard' approaches - e.g., choosing the approximant $x_{n}$ to minimize $\|A x-b\|$ over a subspace $X_{n}$ or Galerkin's method applied to the solution of ill-posed problems.

We observe here, in comparison with Galerkin's method, that the presently proposed algorithm has the inconvenience that the approximating subspaces $X_{n}$ are not specified directly but are computed by applying $A^{*}$ to specifiable subspaces $Y_{n}$, One might, of course, attempt to reverse this by specifying $\left\{z_{1}, \cdots\right\}$ and then solving: $A^{*} y_{j}=z_{j}$ to obtain each $y_{j}$; unfortunately, if the original problem is singular ( $A^{-1}$ unbounded) then each of these equations also involves solution of a singular problem and, in any case, one would be restricted by the necessity of taking $z_{j} \in R\left(A^{*}\right)$.

Some additional freedom can be introduced by varying the inner product. If the original $\langle\cdot, \cdot\rangle$ is replaced by a new inner product $\langle\cdot, \cdot\rangle^{\prime}=\langle\cdot, B \cdot\rangle$, defined in terms of a given positive operator $B$, then the new adjoint $A_{B}^{*}$ is just $B^{-1} A^{*}$ so, now, $A^{*} y_{j}=B z_{j}$. Observe that, if $A$ itself is positive (a point of relevance of the typical imposition of monotonicity conditions in connection with the use of Galerkin's method), then one may take $B=A^{*}(=A)$ and so $y_{j}=z_{j}$ for each $j$. It is easily seen that in this case the present method and Galerkin's method coincide. Needless to say, the $x_{n}$ computed using $\langle\cdot, \cdot\rangle^{\prime}$ is now the best approximant to $x_{*}$ in the sense of the new norm $\|\cdot\|^{\prime}$ and one obtains convergence in $U$ from this only if $\|\cdot\|^{\prime}$ dominates the original norm, i.e., if $B$ has a bounded inverse. Note that even if $A$ is not positive one may replace (1) by

$$
\left(A^{*} A\right) x=b_{*}
$$

with $b_{*}=A^{*} b$ (provided $b \in D_{*}$ ), which will be equivalent to (1) if $R(A)$ is dense so $A^{*}$ is injective. One could then work, instead, with the positive operator $A^{*} A$.

It will be noted that Theorem II proves convergence but gives no direct information as to the rate of convergence. All that can be said about this is given by the characterization of $x_{*}$ as the best approximant in $X_{n}$ to the solution $x_{*}$. If one considers an example with $\left\{z_{1}, z_{2}, \cdots\right\}$ an orthonormal basis of $U$ and $x_{*}=\xi_{1} z_{1}+\xi_{2} z_{2}+\cdots$ selected appropriately (defining $b$ ), it is clear that convergence can be arbitrarily slow. To do better, one must know that, for the particular $\left\{y_{1}, y_{2}, \cdots\right\}$ considered and under whatever 'regularity' properties and a priori bounds as may apply to the specific situation considered, the solution $\hat{x}$ will be approximable in $X_{n}$ with satisfactory accuracy. 
An approach which may be used, on occasion, to provide estimates of convergence rates is to observe that one often has regularity conditions in the form of a stronger existence assertion: not only is $b \in R(A)$ but $b \in R(A C)$, for some operator $C$, so $x_{*}$ has the form: $C u_{*}$ and (1) is replaced by

$$
(A C) u=b .
$$

Applying the same algorithm to solving this gives $\tilde{z}_{j}=(A C)^{*} y_{j}=$ $C^{*} z_{j}$ and (4) gives

$$
\begin{aligned}
\left\langle y_{j}, b\right\rangle & =\left\langle\widetilde{z}_{j}, \hat{u}\right\rangle=\left\langle C^{*} z_{j}, \hat{u}\right\rangle \\
& =\left\langle z_{j}, C \hat{u}\right\rangle
\end{aligned}
$$

so $x_{n}=C u_{n}$ for each $n$. If $C$ is compact (it is typically the embedding operator of one Sobolev space in another), then the convergence of the sequence guaranteed by Theorem II may provide convergence at a usefully estimable (e.g., in terms of $\|\hat{u}\|)$ rate of the sequence $\left\{x_{n}\right\}$. A related approach is used in [3]. Estimation of the convergence rates for some specific applications will appear elsewhere.

6. An application. Let $W$ be the space of functions $w=w(t, \omega)$ for $0 \leqq t \leqq T$ and $\omega \in \Omega$ which satisfy the diffusion equation:

$$
w_{t}=\nabla \cdot p \nabla w
$$

$(p=p(t, \omega)>0)$ and for which

$$
z=w(0, \cdot) \in L_{2}(\Omega), x=\left.w\right|_{\Sigma} \in L_{2}(\Sigma)
$$

(here, $\Omega$ is assumed bounded in $\boldsymbol{R}^{n}$ with $\Sigma=(0, T) \times \partial \Omega$ smooth). Letting $v=w(T, \cdot) \in L_{2}(\Omega)$, one has $v=A x+S z$ with $A: U=L_{2}(\Sigma) \rightarrow V=$ $L_{2}(\Omega)$ and $S: V \rightarrow V$ both bounded linear transformations; $S=S_{T}$ is the solution operator for the pure initial value problem (i.e., for homogeneous Dirichlet conditions) and $A$ gives the effect of the boundary data for homogeneous initial conditions.

Given $z, v$ one can view the situation as a control problem and, in particular, for any $y \in V$ one might seek a null-control: a set of Dirichlet data $x \in U$ for which the solution of (10), (11) vanishes at $T(v=0)$. This amounts to solving the equation: $A x=b(b=-S z)$. It is known (cf, e.g., [10] or [11]) that $R(S) \subset R(A)$ so a solution exists. Indeed, the map: [ $y \mapsto$ nullcontrol $x$ of minimum norm] is a bounded linear map: $V \rightarrow U$, although not directly computationally accessible while $A, S$ are easily available in standard computational practice.

Given $y \in V^{*}(V)$, let $u$ be the solution of the well-posed problem 


$$
-u_{t}=\nabla \cdot p \nabla u,\left.u\right|_{\Sigma}=0, u(T, \cdot)=y \text {. }
$$

Then

$$
\begin{aligned}
\int_{\Omega}(A x) y & =\int_{0}^{T} \int_{\Omega}(w u)_{t} \\
& \left.=\int_{0}^{T} \int_{0}[\nabla \cdot p \nabla w) u-w(\nabla \cdot p \nabla u)\right] \\
& =\int_{\Sigma} p\left[-w_{\nu} u+w u_{\nu}\right]=\int_{\Sigma} p x u_{\nu}
\end{aligned}
$$

Thus $A^{*} y=p u_{\nu}$ where $u_{\nu} \in L_{2}(\Sigma)$ is the normal derivative of the solution of (12).

To compute the approximant $\hat{x}_{n}$ to the desired boundary nullcontrol $\hat{x}$, one would start with $\left\{y_{1}, \cdots, y_{n}\right\}$, solve (12) numerically using each $y_{j}$ as data, and compute each $z_{j}=A^{*} y_{j}=p \partial u_{j} / \partial \nu$. The Gramian $G=\left(\left(\left\langle z_{j}, z_{k}\right\rangle\right)\right)$ can now be obtained by numerical integration over $\Sigma$. To improve the conditioning of the eventual numerical solution of (5), it may be worthwhile to orthonormalize (approximately) $\left\{z_{1}, \cdots, z_{n}\right\}$, reflect the relations of the new basis of $X_{n}$ to the old in re-selecting new $\left\{\widetilde{y}_{1}, \cdots, \widetilde{y}_{n}\right\}$ and then, rather than simply accepting the orthonormalized $\left\{z_{j}\right\}$, re-computing an (almost) orthonormal basis $\left\{\widetilde{z}_{1}, \ldots \widetilde{z}_{n}\right\}$ from $\left\{\widetilde{y}_{1}, \cdots, \widetilde{y}_{n}\right\}$. The term $B=-S z$ is similarly obtained by numerical solution of (10) and (11) with homogeneous boundary data and then the $\left\{\beta_{j}\right\}$ computed by numerical integration over $\Omega$. The resulting system (5) can now be solved to obtain the coefficients $\left\{\xi_{j}\right\}$ and so $\hat{x}_{n}$. Note that the computed $\hat{x}_{n}$ approximates the true $\hat{x}_{n}$ as closely as desired provided the numerical solution of (10), (11) and of (12), the numerical integrations and the solution of (5) are each with sufficient accuracy and, in turn, this will be a good approximant to the solution $\hat{x}$ if $n$ is large enough. An actual computational experiment is described in [2].

\section{REFERENCES}

1. B. C. Buzbee and A. Carasso, On the numerical computation of parabolic problems for preceding times, Math. Comp., 27 (1973), 237-266.

2. W. C. Chewning and T. I. Seidman, A convergent scheme for boundary control of the heat equation, SIAM J. Control, to appear.

3. Joel Franklin, On Tikhonov's method for ill-posed problems, Math. of Comp., 28 (1974), 889-907.

4. J. Hadamard, Lectures on Cauchy's Problem in Linear P.D.E. (sec. 21), 1923, reprinted by Dover Publ., N.Y. (1952).

5. M. A. Krasnoselskii, Solutions of equations involving adjoint operators by successive approximations, Usp. Mat. Nauk, 15 (1960).

6. A. V. Kryanev, The solution of incorrectly posed problems by methods of successive approximations, Soviet Math. Doklady, 14 (1973), 673-676. 
7. R. Lattes and J. L. Lions, The Method of Quasi-Reversibility, Applications to Partial Differential Equations, Amer. Elsevier, N. Y., 1969.

8. M. Z. Nashed and G. Wahba, Regularization and approximation of linear operator equations in reproducing kernel spaces, Bull. Amer. Math. Soc., 80 (1974).

9. W. V. Petryshyn, On the approximation solvability of equations involving A-proper mappings, Bull. Amer. Math. Soc., 81 (1975), 223-312.

10. D. L. Russell, A unified boundary controllability theory for hyperbolic and parabolic partial differential equations, Studies in Appl. Math., 52 (1973), 189-211.

11. T. I. Seidman, Observation and prediction for the heat equation, III, J. Differential Equations, to appear.

12. - Solution of singular equations, II: nonlinear problems, to appear.

13. A. N. Tikhonov, Solution of incorrectly formulated problem and the regularization method., (trans.) Sov. Math. Dokl., 4 (1963), 1036-1038.

Received April 17, 1975.

UNIVERSity OF MARYLAND, BaltimoRe COUNTY 


\section{PACIFIC JOURNAL OF MATHEMATICS}

\section{EDITORS}

RICHARD ARENS (Managing Editor)

University of California

Los Angeles, California 90024

\author{
R. A. Beaumont \\ University of Washington \\ Seattle, Washington 98105
}

\section{J. DugundjI}

Department of Mathematics University of Southern California Los Angeles, California 90007

D. Gilbarg and J. Milgram

Stanford University

Stanford, California 94305

\section{ASSOCIATE EDITORS}
E. F. BECKENBACH
B. H. NeumanN
F. WOLF
K. YoshidA

\section{SUPPORTING INSTITUTIONS}

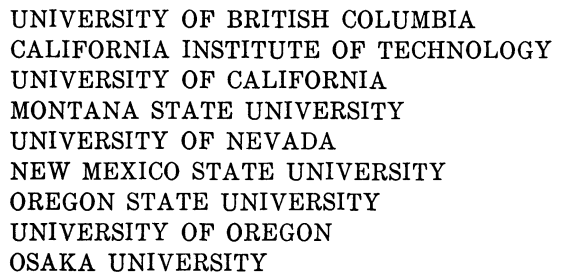

UNIVERSITY OF BRITISH COLUMBIA CALIFORNIA INSTITUTE OF TECHNOLOGY UNIVERSITY OF CALIFORNIA MONTANA STATE UNIVERSITY UNIVERSITY OF NEVADA NEW MEXICO STATE UNIVERSITY OSAKA UNIVERSITY OREGON STATE UNIVERSITY UNIVERSITY OF OREGON

\author{
UNIVERSITY OF SOUTHERN CALIFORNIA \\ STANFORD UNIVERSITY \\ UNIVERSITY OF TOKYO \\ UNIVERSITY OF UTAH \\ WASHINGTON STATE UNIVERSITY \\ UNIVERSITY OF WASHINGTON \\ AMERICAN MATHEMATICAL SOCIETY
}

The Supporting Institutions listed above contribute to the cost of publication of this Journal, but they are not owners or publishers and have no responsibility for its content or policies.

Mathematical papers intended for publication in the Pacific Journal of Mathematics should be in typed form or offset-reproduced, (not dittoed), double spaced with large margins. Please do not use built up fractions in the text of your manuscript. You may however, use them in the displayed equations. Underline Greek letters in red, German in green, and script in blue. The first paragraph or two must be capable of being used separately as a synopsis of the entire paper. Items of the bibliography should not be cited there unless absolutely necessary, in which case they must be identified by author and Journal, rather than by item number. Manuscripts, in triplicate, may be sent to any one of the editors. Please classify according to the scheme of Math. Reviews, Index to Vol. 39. All other communications should be addressed to the managing editor, or Elaine Barth, University of California, Los Angeles, California, 90024.

The Pacific Journal of Mathematics expects the author's institution to pay page charges, and reserves the right to delay publication for nonpayment of charges in case of financial emergency.

100 reprints are provided free for each article, only if page charges have been substantially paid. Additional copies may be obtained at cost in multiples of 50 .

The Pacific Journal of Mathematics is issued monthly as of January 1966. Regular subscription rate: $\$ 72.00$ a year $(6$ Vols., 12 issues). Special rate: $\$ 36.00$ a year to individual members of supporting institutions.

Subscriptions, orders for back numbers, and changes of address should be sent to Pacific Journal of Mathematics, 103 Highland Boulevard, Berkeley, California, 94708.

PUBLISHED BY PACIFIC JOURNAL OF MATHEMATICS, A NON-PROFIT CORPORATION

Printed at Kokusai Bunken Insatsusha (International Academic Printing Co., Ltd.), 8-8, 3-chome, Takadanobaba, Shinjuku-ku, Tokyo 160, Japan. 


\section{Pacific Journal of Mathematics}

\section{Vol. 61, No. 2 December, 1975}

Graham Donald Allen, Francis Joseph Narcowich and James Patrick Williams, An operator version of a theorem of Kolmogorov .......................

Joel Hilary Anderson and Ciprian Foias, Properties which normal operators share with normal derivations and related operators . . . . . . . . . . . . . . . . . . . . .

Constantin Gelu Apostol and Norberto Salinas, Nilpotent approximations and

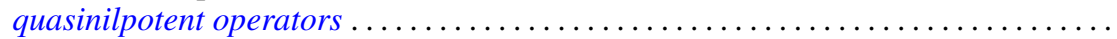

James M. Briggs, Jr., Finitely generated ideals in regular $F$-algebras . . . . . . . . . . .

Frank Benjamin Cannonito and Ronald Wallace Gatterdam, The word problem and power problem in 1-relator groups are primitive recursive ..................

Clifton Earle Corzatt, Permutation polynomials over the rational numbers ...........

L. S. Dube, An inversion of the $S_{2}$ transform for generalized functions . . . . . . . . . . William Richard Emerson, Averaging strongly subadditive set functions in unimodular

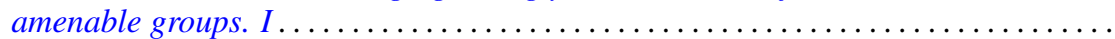

Barry J. Gardner, Semi-simple radical classes of algebras and attainability of

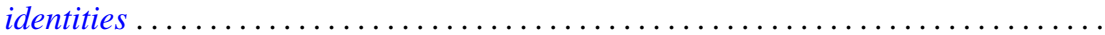

Irving Leonard Glicksberg, Removable discontinuities of A-holomorphic functions ....

Fred Halpern, Transfer theorems for topological structures . . . . . . . . . . . . . . .

H. B. Hamilton, T. E. Nordahl and Takayuki Tamura, Commutative cancellative

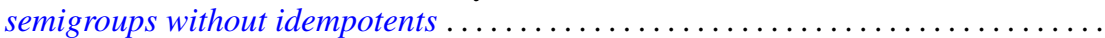

Melvin Hochster, An obstruction to lifting cyclic modules .....................

Alistair H. Lachlan, Theories with a finite number of models in an uncountable power

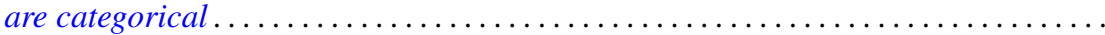

Kjeld Laursen, Continuity of linear maps from $C^{*}$-algebras . . . . . . . . . . . . .

Tsai Sheng Liu, Oscillation of even order differential equations with deviating arguments ....

Jorge Martinez, Doubling chains, singular elements and hyper- $Z$

Mehdi Radjabalipour and Heydar Radjavi, On the geometry of num Thomas I. Seidman, The solution of singular equations, I. Linear equations in Hilbert

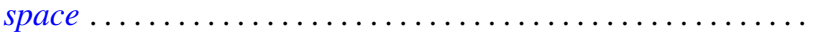

R. James Tomkins, Properties of martingale-like sequences ......

Alfons Van Daele, A Radon Nikodým theorem for weights on von Neumann

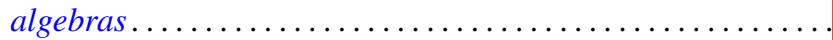

Kenneth S. Williams, On Euler's criterion for quintic nonresidues

Scott Andrew Wolpert, Noncompleteness of the Weil-Petersson metric for Teichmüller

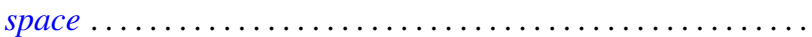

Volker Wrobel, Some generalizations of Schauder's theorem in locally convex

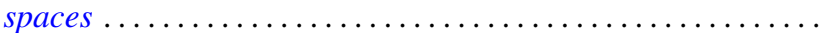

Kelly Denis McKennon, Corrections to: "Multipliers of type $(p, p)$ "; "Multipliers of type $(p, p)$ and multipliers of the group $L_{p}$-algebras"; "Multipliers and the

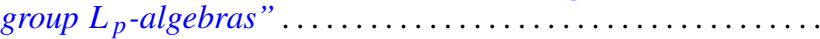

Andrew M. W. Glass, W. Charles (Wilbur) Holland Jr. and Stephen H. McCleary, Correction to: " $a *$-closures to completely distributive lattice-ordered

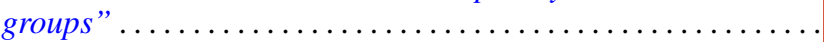

Zvi Arad and George Isaac Glauberman, Correction to: "A characteristic subgroup of

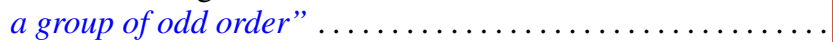

Roger W. Barnard and John Lawson Lewis, Correction to: "Subordination theorems

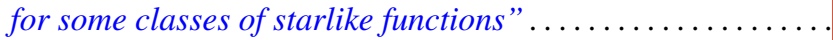

